\title{
Enhanced Recovery Program (ERP) versus Traditional care after Elective Left Side Colorectal Cancer Surgery \\ Ahmad Aboelkassem Ibrahim ${ }^{2}$, Reda M. Moustafa ${ }^{1}$, Ashraf Abdulmoghni Moustafa ${ }^{1}$, Saleem EL-Rabaa ${ }^{2}$, Yahya Salama ${ }^{2}$ \\ ${ }^{1}$ Department of General Surgery, Faculty of Medicine - Ain Shams University - Egypt \\ ${ }^{2}$ Colorectal Department, Kettering General Hospital - United Kingdom \\ Corresponding author: Ahmad M. Aboelkassem Ibrahim; Email: draboelkassem@ hotmail.com
}

\begin{abstract}
Aims: We aimed to study the effect of ERAS protocol after left side colorectal cancer surgery in comparison with patients were subjected to traditional care. Methods: Retrospective study comparing 2 groups of patients who underwent elective left side colorectal cancer surgery. Group A: 25 patients admitted between November 2014 and April 2015 subjected to ERAS, and Group B: 25 patients admitted between January 2008 and August 2008 received traditional care. Both groups were consecutive cases. The notes were examined to determine the following outcome measures: Short-term morbidity, Length of stay, 30 days Readmission rate and Mortality. Results: 25 patients with ERAS matched with 25 traditional recovery cases for baseline demographics. $96 \%$ started oral fluids on D1 in group A compared to $8 \%$ in group B, $96 \%$ in group. A tolerated full diet on D6 compared to $72 \%$ in group B. Bowel movements by D5 was group A $92 \%$ compared to only $36 \%$ in group B. Total LOS mean for group A was 7 days vs 12.48 in group $B(p=0.005)$. Complications occurred less frequently in group A compared to group B (anastomotic leak with $8 \%(n=2)$ in group A versus $16 \%(n=4)$ in group $B$, prolonged ileus with $8 \%(n=2)$ in group A versus $16 \%(n=4)$ in group $B(p=0.663)$. Mobility on D1 $40 \%(\mathrm{n}=10)$ in group A was an independent factor which decreased LOS, as all stayed $<5$ days $(\mathrm{p}=0.002)$ and developed less complications $(4 \%(\mathrm{p}=0.027))$. Conclusion: ERAS didn't affect complications rate significantly following left side colorectal surgery, however reduced LOS as it improved tolerating oral intake and bowel movement. Mobility D1 can be independent pridector of reduced both complications and LOS and should be encouraged for all patients.
\end{abstract}

Key words: inhanced recovery program, traditional care, colorectal cancer surgery

\section{INTRODUCTION}

Colorectal cancer (CRC) continues to be a leading cause of cancer associated with significant morbidity and mortality in the United States (US) and throughout the world. Globally, CRC is the third most common cancer in men and the second in women ${ }^{(\mathbf{1})}$.

Conventional colorectal operations for malignant disease are usually associated with extensive preoperative preparation, significant surgical stress, and prolonged recovery of gastrointestinal function and the body as a whole. An excessive stress response may predispose the patient to an increased risk of cardiovascular and cerebrovascular complications, nutrient malabsorption, and delayed convalescence ${ }^{(2)}$.

Many surgeons believe that left colectomy (LC), which is often more technically challenging and requires a colo-colic or colorectal anastomosis, has a significantly higher incidence of anastomotic leakage, wound infection, overall complication rate, and longer length of hospital stay (LOS) compared to right colectomy (RC), which utilizes an ileocolic anastomosis. Therefore, $\mathrm{RC}$ is generally believed to be a simpler operation with better outcomes than LC. Left colectomy had higher intraoperative complications and total hospital stay than right colectomy ${ }^{(3)}$.
Colorectal surgery has been associated with complication rates ranging from $10 \%$ to $20 \%$ and mean postoperative hospital stays from 6 to 10 days. The financial burden imposed on health care systems due to prolonged hospital stay after colorectal surgery can be significant. In an effort to improve postoperative outcomes in patients undergoing colorectal surgery, enhanced recovery after surgery (ERAS) programs has been designed and evaluated ${ }^{(4)}$.

Kehlet et al. ${ }^{(5)}$ were the first to describe in detail a specific protocol called "fast-track" or "enhanced recovery after surgery" protocol which had the potential to reduce hospital stay to a mean of 4 days, largely as a result of better understanding of postoperative physiology and advances in the field of health care in the last decade. The aim is to attenuate the surgical stress response, accelerate recovery, decrease complications, minimize hospital stay, and ultimately reduce health costs without compromising patients' safety ${ }^{(6)}$.

ERAS interventions focus on those key factors that usually keep patients in hospital and make them dependent on drugs and specialist assistance following uncomplicated surgery, namely the need for parenteral analgesia, the administration of intravenous fluids and confinement to bed. Pillars of 
ERAS protocols cover all the peri-operative phases (pre-operative, intra-operative and post-operative) by removing or decreasing the influence of such factors and promoting good habits that favors the recovery of physiological functions. Therefore, they avoid mechanical bowel preparations (MBPs) and preoperative fasting before surgery and administer high carbohydrate meals until few hours before the operation; they limit the administration of fluids tailoring them to the real patient's necessities during surgery; they encourage the resumption of an oral diet and early mobilization after surgery as well as they decrease the use of regular opioids using pain killers with less impact on the gut function ${ }^{(7)}$.

The introduction of postoperative care pathways over the last decade has resulted in a reduction in postoperative length of stay with acceptable readmission rates. This paradigm shift has occurred at a time when advanced laparoscopic techniques are being applied to a variety of colorectal conditions. It has been shown that laparoscopy reduces length of stay. Despite the cost associated with minimally invasive equipment, the overall benefit in relation to bed days saved has been documented. It is unclear whether combining ERPs and laparoscopic techniques further reduces the postoperative stay given the fact that the initial work described by Basse et al. ${ }^{(8)}$ was in the setting of open colorectal surgery ${ }^{(9)}$.

However, it is still controversial if colorectal multimodal rehabilitation programs offer substantial benefits to patients. The argument is that fast-track protocols increase complication and readmission rate sufficiently that overall they do not reduce health costs or total hospital stay. Additionally, complications of fast-track patients are recognized relatively late with possibly significant consequences ${ }^{(6)}$.

Since their introduction ERAS protocols faced large resistances because they targeted diffuse and time-validated clinical practices. These were mostly based on tradition, personal experiences, and surgical teaching that helped their historical perpetration. However, the growing amount of data available has showed now how such practices were not necessary or contributed to the adverse effects of the surgical trauma. The most immediate and visible effect of ERAS introduction is a significant shortening of the length of stay (LOS) in hospital and therefore a better redistribution of the available resources. Nowadays ERAS is routine in large university hospitals and is also spreading to district general hospitals with special interests in colorectal operations ${ }^{(10)}$.

\section{PATIENTS AND METHODS}

This is a comparative study included 50 patients admitted to Kettering General Hospital who underwent an elective left side colorectal cancer surgery divided into two groups: Group A: 25 patients were subjected to enhanced recovery pathway after elective left side colorectal cancer surgery admitted to Kettering General Hospital between November 2014 and April 2015. This group consisted of 12 females $(48 \%)$ and 13 males $(52 \%)$ with a range of age 46-87 years with a mean of 65.6, and Group B: 25 patients were subjected to traditional care after elective left side colorectal cancer surgery admitted to Kettering General Hospital between January 2008 and August 2008. This group consisted of 6 females $(24.0 \%)$ and 19 males $(76.0 \%)$ with a range of age 50-85 years with a mean of 68.16.

\section{Enhanced Recovery Program:}

Preoperative: Preoperative counseling with a colorectal nurse specialist, Admission in the morning of the day of surgery, Decrease fasting hours (6 hours solid, 2 hours clear fluids), Carbohydrate loading with carbohydrate rich fluid 2 hours before surgery, Bowel preparation: (Phosphate enema early morning on the day of the operation for patients who had left hemi-colectomy and sigmoid colectomy and patients had Anterior Resection were subjected to full bowel preparation (CitraFleet $\AA$; sodium picosulfate powder $10 \mathrm{mg} /$ sachet, one day before surgery; 1 sachet should be reconstituted in $150 \mathrm{ml}$ of cold water; patients should take 1 sachet before 8 am and a $2^{\text {nd }}$ sachet 6-8 hours later.), Venous Thrombo-Embolism (VTE) prophylaxis: All patients- unless contraindicated- had mechanical prophylaxis; thromboembolic deterrent stockings and intermittent pneumatic compression devices, in addition to chemical prophylaxis; once daily Enoxaparin $40 \mathrm{mg}$ subcutaneous injection starting 6 hours after surgery and Antibiotics prophylaxis: 
Intravenous antibiotics (according to hospital policy) within 60 minutes before surgery. The study was approved by the Ethics Board of Ain Shams University and an informed written consent was taken from each participant in the study.

Intra-operative: Regional block used in addition to general anaesthesia during surgery, Short-acting induction agents such as propofol combined with a short-acting opioid like fentanyl and short-acting muscle relaxants titrated using neuromuscular monitoring. Laparoscopic approach was the standard unless contraindicated or converted due difficulties (Laparoscopic 52\%, open $\mathbf{4 8 \%}$ ), Prevent hypothermia (Bair Hugger system), Goal directed intravenous fluid therapy (using oesophageal Doppler) and avoidance of the routine usage of drains and Nasogastric tubes (Drains in 76\%, no NG tubes $100 \%$ ).

Post-operative: documented in Enhanced recovery Grid: Encouraging postoperative early mobilization, Early oral nutrition, Early removal of all tubes including (drains, epidural catheter and urinary catheter) and Analgesia: According to the hospital protocol.

Traditional care: Preoperative: Admission one night before surgery, Full bowel preparation for all patients, Fasting 8 hours before the operation, VTE prophylaxis and Antibiotic prophylaxis (same as group A).

Intra-operative: Liberal Intra-venous fluid regimen, Usage of general anesthesia without regional blocks and Routine use of drains and nasogastric tubes.

Post-operative: Nil by mouth till bowel movement (passing flatus), Usage of Patient control analgesia (PCA) opiate analgesics + regular paracetamol \pm regular Ibuprofen and Mobility as tolerated by patients.

Primary outcome: Length of total postoperative stay expressed as total days spent in the hospital including possible 30 days readmissions.
Secondary outcomes: Short-term morbidity including anastomotic leak and general postoperative complications (bowel obstruction, prolonged ileus, abscess formation, wound infection, and pulmonary embolism), Readmission rate and Mortality.

Data collection: Data were collected from patients' records and medical files.

Inclusion criteria: Age: more than 18 years. Gender: both sexes. Elective left side colorectal cancer surgery +/- stoma. Postoperative level 1(ward) or 2 (High Dependency Unit) care.

Exclusion criteria: Post-operative level 3 care (Intensive Therapy Unit). Patient refusal. Other colorectal surgeries rather than elective left side colorectal cancer surgery. Patients without easy access back to the hospital.

Discharge criteria: Adequate pain control with simple oral analgesics. Tolerating full diet oral intake. Opening bowel/Passing flatus. Dry wound.

\section{RESULTS}

The highest comorbidity was hypertension in group A and B 24\% $(n=6)$ and $44 \%(n=11)$ respectively, other comorbid diseases included Diabetes mellitus (DM) $16 \%(n=4)$ were equal in both groups, Ischemic heart disease (IHD) $12 \%(\mathrm{n}=3)$ in group $\mathrm{A}$ and $16 \%$ $(n=4)$ in group $B$, Chronic obstructive pulmonary disease (COPD) $8 \%(n=2)$ in group A and $4 \%(n=1)$ in group $\mathrm{B}$, one patient had prostate cancer and other one had chronic kidney disease in group A and one patient had aortic valve stenosis in group B. There was no statistically significant difference between both groups according to comorbidities.

All patients in group B had full bowel preparation $100 \%(\mathrm{n}=25)$ compared to $56 \%(\mathrm{n}=14)$ in group A while the rest of group A patients had only enema preoperatively $44 \%(n=11)$ compared to $0 \%$ in group $\mathrm{B}$. There was a highly statistically significant difference between both groups according to bowel preparation.

In group A $52 \% \quad(n=13)$ underwent laparoscopic surgery and $48 \%(\mathrm{n}=12)$ underwent open procedure (either from the start or converted to 
open because of difficulties), compared to Group B $12 \%(n=3)$ underwent laparoscopic surgery versus $88 \%$ open surgery (beginning of the era of laparoscopic colorectal surgery in our unit 2007). There was a statistically significant difference between groups according to the approach of operation. The tumors in both groups were left sided and rectal. Equal number of patients in both groups underwent Anterior Resection (AR) 60\% ( $\mathrm{n}=15$ ), while $32 \%(\mathrm{n}=8)$ in group A underwent sigmoid colectomy versus $36 \%(\mathrm{n}=9)$ in group $\mathrm{B}$, and $8 \%$ $(\mathrm{n}=2)$ in group A underwent left hemi-colectomy versus $4 \% \quad(n=1)$ in group B. There was no statistically significant difference between groups according to type of operation.

As regards postoperative analgesia $72 \%$ $(\mathrm{n}=18)$ in group A had Epidural analgesia compared to $56 \%(n=14)$ in group $B$, while $44 \%(n=11)$ in group $\mathrm{B}$ had intravenous Morphine patient-controlled analgesia (PCA) versus $28 \%(n=7)$ in group A. There was no statistically significant difference between groups according to methods of pain control.

\section{Mobility distribution in group $\mathrm{A}$ :}

Only $40 \%(n=10)$ of patients were compliant with early mobilization in day 1 , the number increased to $72 \%(\mathrm{n}=18)$ in day 2 , and $88 \%(\mathrm{n}=22)$ of patients were independently mobile on day 3 , while $12 \%(n=3)$ were immobile till day 4 due to inadequate pain control.

In group A $96 \%(n=24)$ of the patients started oral intake on day 1 (D1), $20 \%$ out of them were 2 hours post-operative; this is compared to group B where all patients were NPO on day 0 , only $8 \%(n=2)$ started oral fluids on D1. There was a highly statistically significant difference between groups regarding postoperative nutrition. Bowel movements by D5 was group A 92\% compared to only $36 \%$ in group B.

The postoperative complications included: Gastrointestinal (GIT) complications 36\% in group B compared to $16 \%$ in group A (anastomotic leak with $16 \%$ $(\mathrm{n}=4)$ in group $\mathrm{B}$ versus $8 \%(\mathrm{n}=2)$ in group A -all patients in both groups underwent low anterior resection and were subjected to full mechanical bowel preparation-, prolonged ileus with $16 \%(\mathrm{n}=4)$ in group $\mathrm{B}$ versus $8 \%(\mathrm{n}=2)$ in group $\mathrm{B}$ and only 1 patient had high output stoma in group B). Other complications included: pneumonia with $16 \%(\mathrm{n}=4)$ in group A versus $8 \%(n=2)$ in group $B$, one patient in group A had urinary tract infection (UTI) and another one had wound dehiscence while $12 \%(n=3)$ in group B had wound infection compared to $0 \%$ in group A. There was no statistically significant difference between groups regarding the complications.

Both groups had equal 30 days readmissions $12 \%(n=3)$. There was no statistically significant difference between groups according to readmission.

As regards total LOS after 30 days readmissions there was a statistically significant difference between both groups, as the range of total LOS in group A remained as 2-18 days with a mean \pm $\mathrm{SD}$ of $7.060 \pm 4.54$; this is 5.42 days shorter stay than the mean \pm SD of $12.48 \pm 6.89$ in group $B$ while the range was 5-38 days. (Table 1)

Table (1): Comparison between groups according to total length of stay.

\begin{tabular}{|l|c|c|c|c|}
\cline { 1 - 3 } Total Length of stay (day) & $\begin{array}{c}\text { Group A } \\
(\mathbf{N = 2 5 )}\end{array}$ & $\begin{array}{c}\text { Group B } \\
(\mathbf{N = 2 5 )}\end{array}$ & x2 & p-value \\
\cline { 1 - 3 } Mean \pm SD & $7.06 \pm 4.54$ & $12.48 \pm 6.89$ & \multirow{2}{*}{8.743} & \multirow{2}{*}{0.005} \\
\hline Range & $2-18$ & $5-38$ & \\
\hline
\end{tabular}

There was a statistically significant relation between complications according to gender (more in males), approach of operation (more in open surgery), stoma, NG tube, mobility D1, mobility D3, date of full diet, date of bowel movement and total length of stay category in group A. (fig 1)

There was a statistically significant relation between total length of stay and approach of operation, pain D1, mobilityD1, mobility D3, and readmission in the group A. (fig 2)

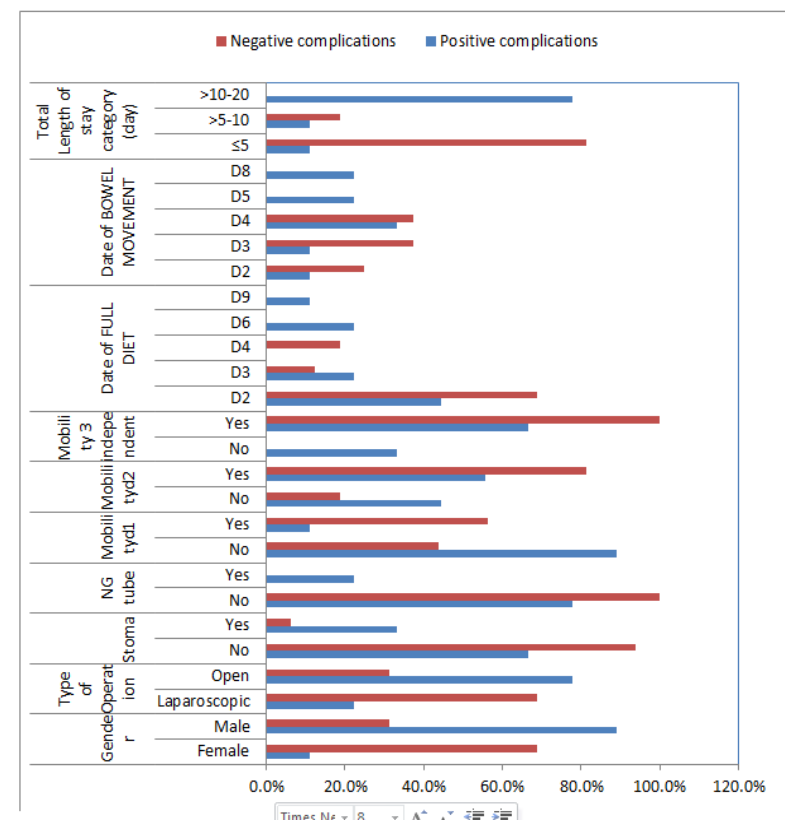

Figure (1): Bar chart between complications and all parameters in Group A. 


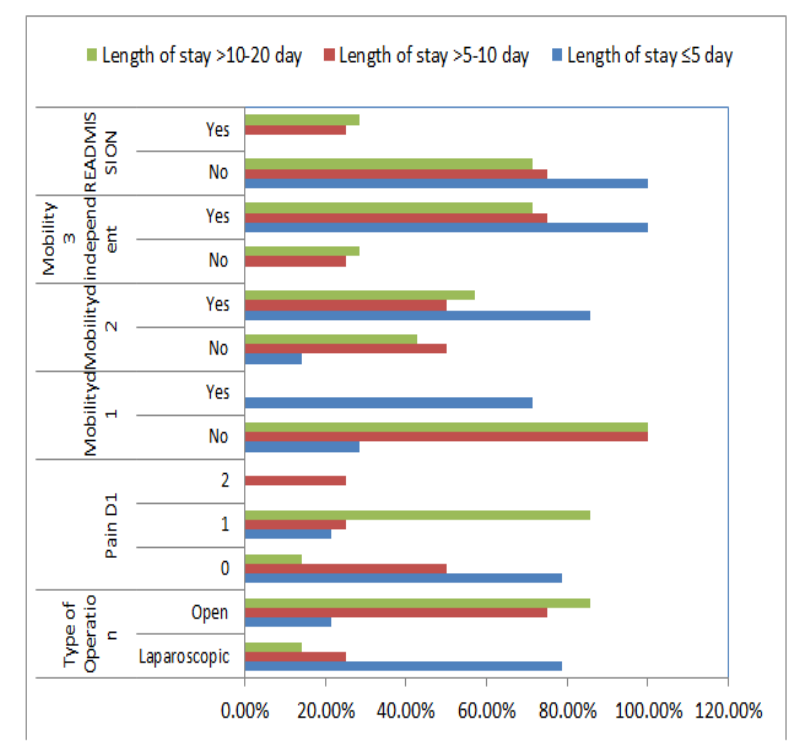

Figure (2): Bar chart between total length of stay and all parameters in Group A.

There was a Positive significant correlation between total length of stay and date of full diet, date of bowel movement (fig 3).
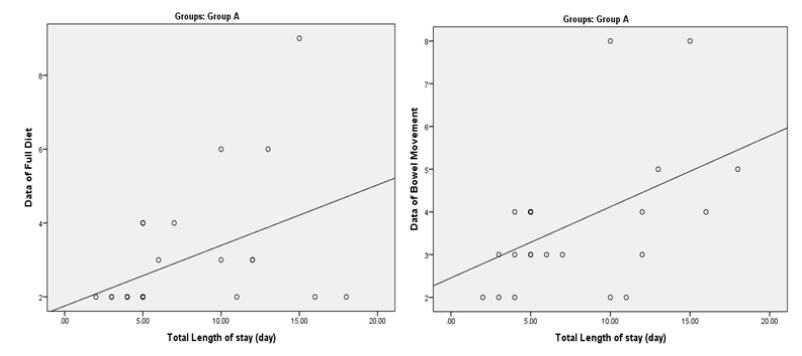

\section{DISCUSSION}

Fast-track protocols or enhanced recovery programs are treatment protocols made with adherence to evidence-based principles. Traditionally, studies comparing different treatment protocols have been difficult to carry out, and their results are difficult to interpret uniformly. This difficulty is increased when surgical procedures are involved because of the number of variables involved and the difficulty in standardizing outcomes and complications. Results of clinical studies on patients in fast-track colon surgery protocols have been reported since Kehlet et al. ${ }^{(5)}$ introduced the concept.

This study has been conducted to evaluate the concept of early oral feeding, thoracic epidural analgesia to control postoperative pain and enforced mobilization protocol and integral parts of enhanced recovery program compared to delayed oral feeding, conventional analgesia and mobilization as tolerated by patients in terms of primary outcome parameters as hospital stay, and surgery related immediate postoperative complications in patients undergoing elective colorectal surgery.

As regard age and gender, in this study, no relation was observed between age and gender and reduced LOS, however, males showed significant increase risk of post-operative complications compared to females and the traditional care groups. Delany et al. (11), found that early post-operative feeding is particularly beneficial for patients younger than 70 years old. Difronzo et al. ${ }^{(12)}$ found no significant differences were between ages .Furthermore, they found that males were more associated with early post-operative feeding intolerance in comparison with females. Also, Petrelliet et al. ${ }^{(13)}$ did not find male sex to induce an effect on whether would tolerate early oral feeding.

Svanfeld et al. (14) showed that patients receiving preoperative oral carbohydrate drinks have less muscle loss and better whole-body protein balance after major abdominal surgery; it has also been shown that oral carbohydrate loading was associated with shorter hospital stays. A Cochrane Database systematic review showed that a 2-h preoperative fasting for clear liquids and a 6-h fast for solids were not associated with any increase in complications ${ }^{(\mathbf{1 5})}$, which is in agreement with those of our study.

As regards Mechanical bowel preparation, this study showed that full bowel preparation didn't affect the rate of post-operative complications including anastomotic leak. Similar results were also shown in a meta-analysis that bowel cleansing by the means of mechanical bowel preparation has not been demonstrated to reduce postoperative complication rates in randomized clinical trials ${ }^{(\mathbf{1 6})}$. Furthermore, RCTs evaluating mechanical bowel preparation in elective colorectal surgery either showed no benefit or harmful effects of mechanical bowel cleansing.

Tokuhara et al. ${ }^{(17)}$ have shown similar results to our study findings that the use of laparoscopic surgery is associated with shorter hospital stay and early ambulation, as 11 out of the 13 patients who underwent laparoscopic surgery in the study group had hospital stay $\leq 5$ days. Even though laparoscopic surgery is advisable wherever possible. It is unclear whether the laparoscopic approach further improves on the short-term recovery benefits 
already seen with multimodal rehabilitation programs; benefits have been reported in some studies like those conducted by Kennedy et al. ${ }^{(18)}$ but not in others as shown by Lyon $\boldsymbol{e t} \boldsymbol{a l} \mathbf{.}^{(\mathbf{1 9})}$.

Pavlin et al. ${ }^{(20)}$ have shown in their study that pain remains the most common reason for delaying discharge after ambulatory surgery. Obtaining a subjective feeling of pain relief has been associated with faster mobilization and early ambulation. It even facilitates early discharge. The multimodal approach to analgesia offers better pain relief in the immediate postoperative period, a combination of local and regional anaesthesia, our study showed similar results as 14 patients in the study group had pain score 0 in day 1,11 out of them had no complications and LOS $\leq 5$ days.

Ileus or a lack of propulsive movement of the intestine is a very distressing post-operative complication in patients undergoing gastrointestinal surgery. Melnyk et al. ${ }^{\text {(21) }}$ have demonstrated in their study that the use of mechanical bowel cleansing leads to increased incidence of ileus. Lubawski $\boldsymbol{e t}$ al. ${ }^{(22)}$ have, in their study on post-operative ileus, shown that early feeding plays a role in reducing the incidence of ileus.

Bed rest should be discouraged after elective surgery. The patient should be encouraged to move around first with help and then independently. Henriksen et al. ${ }^{(23)}$ have demonstrated that enforced ambulation is significantly associated with decreased hospital stay. Our study results showed a significant reduction in both LOS and complications in the group mobilized in day $1(40 \% \mathrm{n}=10)$ within the study group; as all patients in that group had no complications and LOS $\leq 5$ days. Also, there was a significant relation between reduction of both LOS and complications, and the independent mobility on day 3 .

Several studies and meta-analyses have shown that patients who have had gastrointestinal anastomoses need not to be kept nil by mouth for prolonged periods ${ }^{(24)}$. Andersen et al. ${ }^{(25)}$ have also demonstrated in their study that early enteral nutrition does not increase the incidence of anastomotic dehiscence. When considering the overall costbenefit of enteral nutrition which is cheaper, the lower incidence of infectious complications and maintenance of lean body mass seem reasonable to offer a trial of early enteral nutrition, our study showed similar results as there was a significant relation between early oral intake $(96 \%$ tolerated oral intake by Day 1) and reduced both LOS and post-operative complications in the study group.

Basse et al ${ }^{(8)}$ demonstrated that an early oral feeding within 24 hours after gastrointestinal surgery is well tolerated, safe and plays an important role to enhance recovery and outcome. Seenu and Goel et $\boldsymbol{a l} .{ }^{(30)}$ showed that early oral feeding after elective colorectal surgery is safe and can be tolerated by most patients.

As regard early post-operative complications, this study justify that early postoperative feeding in colorectal surgery didn't jeopardize the recently performed colonic anastomosis, as there were only two cases of clinical anastomotic leak in the early fed group compared to four cases in the delayed oral feeding group. Difronzo et al. ${ }^{(12)}$ also found no incidence of anastomotic leak in 200 patients studied for early post-operative feeding after open colon resection.

Also, this study showed that early oral feeding didn't increase the risk of post-operative ileus as only 2 patients in the study group suffered from prolonged ileus and need NG tubes compared to 4 patients in the traditional group.

As regard length of hospital stay, the main achievement of early post-operative feeding was the considerable reduction in hospital stay. In the present study, the length of hospital stay was significantly shorter among patients of the study group with a mean post-operative hospital stay of 7 days, while with the traditional group the mean was 12.48 days; this was 5.48 days shorter in the study group with high statistical significant difference between the groups $\mathrm{P}<0.005$. This was confirmed the beneficial effect of enhanced recovery program after surgery in reducing length of hospital stay with its physical, psychological and economic benefits.

Waiter et al. ${ }^{(26)}$, reported median length of hospital stay 5 days, range 4-7 days in fast track patients, versus 7 days, range (6-8 days) in non-fast track patients) .However Hjort et al. ${ }^{(29)}$ had the same results of median hospital stay of 2 days versus 8 days in fast track versus conventional care with less cost and more patients satisfaction with also earlier resumption of normal activities, but they reported more frequent readmissions, 5 patients in fast track versus one patient in conventional care. 
Kehlet et al. (5) reported median postoperative hospital stay of 2 (range 2-6) days, in 18 patients underwent elective sigmoid resection. Patients being mobilized for a median of 5 hours on the second postoperative day (24-48 h) and for 10 hours on the third day (48-72 h). There were no medical or surgical complications during 30 days of follow-up, except for two patients who suffered post spinal headache. Difronzo et al. ${ }^{(12)}$, found on consecutive case series on 200 consecutive patients undergoing elective open colon resection that early postoperative feeding in safe and effective, and produces a brief hospital stay compared with patients fed by traditional means.

As regards the readmission rate, in this study, three patients from each group were readmitted after discharging due to different causes. Thus, readmission rate was equal in both groups despite of early discharge in the study group patients. This observation also was demonstrated by Nanavati et al. ${ }^{(27)}$ in their study.

As regards mortality, in this study no mortality was reported in both groups. This confirmed that ERP is not associated with increased mortality. Proske et al. in $2005^{(28)}$ found that there were no significant differences in mortality between early oral feeding and delayed oral feeding after intestinal anastomosis. Other studies confirmed the reduction in mortality rate with early oral feeding.

Finally, the results of the presented study seem to agree with those previously mentioned in terms of hospital stay, general and local complications.

\section{CONCLUSION}

From our study we concluded that: Enhanced recovery pathway is safe and tolerable practice after left sided colorectal cancer surgery with no increase in the post-operative complications. Mechanical bowel preparation didn't affect neither the rate of post-operative anastomotic leak nor other complications.

\section{RECOMMENDATIONS}

Further studies are required to assess the effect of fast track surgery on elderly patients. Further investigations are also needed to assess the role of minimally invasive surgery within the setting of enhanced recovery pathway in reducing the LOS.

\section{REFERENCES}

1. Douaiher J, Ravipati A, Grams B, Chowdhury S, Alatise $O$ and Are C (2017): Colorectal cancer-global burden, trends, and geographical variations. J Surg Oncol., 115:619-630.

2. Ren L, Zhu D, Wei Y et al. (2012): "Enhanced Recovery After Surgery (ERAS) Program Attenuates Stress and Accelerates Recovery in Patients After Radical Resection for Colorectal Cancer: A Prospective Randomized Controlled Trial", World J Surg., 36:407-414.

3. Masoomi H, Buchberg B, Dang $\mathbf{P}$, Carmichael JC, Mills $\mathbf{S}$ and Stamos MJ (2011): Outcomes of right vs. left colectomy for colon cancer. J Gastrointest Surg., 15(11):2023-8.

4. Eskicioglu C, Forbes S, Aarts $M$ et al. (2009): "Enhanced Recovery after Surgery (ERAS) Programs for Patients Having Colorectal Surgery: A Meta-analysis of Randomized Trials", J Gastrointest Surg., 13:2321-2329.

5. Kehlet H (1999): Acute pain control and accelerated post-operative recovery. Surgical Clin N Am., 79:431-43.

6. Gouvas N, Tan E, Windsor A et al. (2009): " Fast-track vs standard care in colorectal surgery: a meta-analysis update". Int $\mathrm{J}$ Colorectal Dis., 24:1119-1131.

7. Varadhan KK, Lobo DN and Ljungqvist $O$ (2010): "Enhanced recovery after surgery: the future of improving surgical care". Crit Care Clin., 26:527-47.

8. Basse $\mathbf{L}$, Lysgaard-Madsen $\mathbf{J}$ and Kehlet $\mathbf{H}$ (2011): Normal gastrointestinal transit after colonic resection with epidural analgesia, enteral oral nutrition, and laxative. Br J Surg., 88: $1498-500$.

9. Al Chalabi H, Kavanagh DO, Hassan L, Donnell KO, Nugent E, Andrews E, Keane FB, O'Riordain DS, Miller A and Neary $P$ (2010): The benefit of an enhanced recovery programme following elective laparoscopic sigmoid colectomy. International journal of colorectal disease, 25(6):761-6.

10. Gravante $G$ and Elmussareh $M$ (2012): "Enhanced recovery for colorectal surgery: Practical hints, results and future challenges". World J Gastrointest Surg., 4(8): 190-198. 
11. Delaney CP, Fazio VW, Senagor AJ, Robinson B, Halverson AL and Ramzi FH (2001): Fast track post operative management protocol for patients with high co morbidity undergoing complex abdominal and pelvic colorectal surgery. Br J Surg., 88: 1533-8.

12. Difronzo LA, Cymerman $\mathbf{J}$ and $\mathbf{O}^{\prime}$ Connel TX (2006): Factors affecting early post operative feeding following elective open colon resection. Arch Surg., 134: 941-6.

13. Petrelli NJ, Cheng C, Driscoll D, Miguel A and Bigas R (2001): Early post operative oral feeding after colectomy: An analysis of factors that may predict failure. Ann SurgOnco., 8: 796-800.

14. Svanfeldt M, Thorell A, Hausel J, Soop M, Rooyackers $O$, Nygren $J$ and Ljungqvist $O$ (2007): Randomized clinical trial of the effect of preoperative oral carbohydrate treatment on postoperative whole-body protein and glucose kinetics. British J Surg., 94(11):1342-50.

15. Brady M, Kinn $S$ and Stuart $P$ (2003): Preoperative fasting for adults to prevent perioperative complications: Cochrane Database Syst Rev., CD004423.

16. Buscher $\mathbf{P}$, Mermillod $\mathbf{B}$, Gervaz $\mathbf{P}$ and Morel P (2004): Mechanical bowel preparation for elective colorectal surgery: a meta-analysis. Arch Surg., 139(12):1359-64.

17. Tokuhara K, Nakatani $\mathbf{K}$, Ueyama $\mathbf{Y}$, Yoshioka K and Kon M (2016): Short-and long-term outcomes of laparoscopic surgery for colorectal cancer in the elderly: a prospective cohort study. International Journal of Surgery, 27:66-71.

18. Kennedy RH and King PM (2004): Laparoscopic surgery for colorectal cancer. Ann R Coll Surg Engl., 86(4):298-99.

19. Lyon A, Payne CJ and Mackay G (2012): Enhanced recovery program in colorectal surgery: Does one size fit all? World J Gastroenterol., 18(40):5661-5663.

20. Pavlin DJ, Chen C, Penaloza DA, Polissar NL and Buckley FP (2002): Pain as a factor complicating recovery and discharge after ambulatory surgery. Anesth Analg., 95(3):62734.

21. Melnyk M, Casey R and Koupparis A (2011): Enhanced recovery after surgery (ERAS) protocols: Time to change practice? Can Urol Assoc J ., 5(5):342-348.
22. Lubawski $J$ and Saclarides $T$ (2008): Postoperative ileus: strategies for reduction. Ther Clin Risk Manag., 4(5): 913-7.

23. Henriksen MG, Hansen HV and Hessov I (2002): Early oral nutrition after elective colorectal surgery: influence of balanced analgesia and enforced mobilization. Nutrition, 263,7 .

24. Lewis SJ, Egger M, Sylvester $\mathbf{P}$ and Thomas SA (2001): Early enteral feeding versus "nil by mouth" after gastrointestinal surgery: systematic review and meta-analysis of controlled trials. BMJ., 323(7316):773.

25. Andersen HK, Lewis SJ and Thomas S (2006): Early enteral nutrition within $24 \mathrm{~h}$ of colorectal surgery versus later commencement of feeding for postoperative complications. Cochrane Database Syst Rev., https://www.ncbi.nlm.nih.gov/pubmed/170541 96

26. Waiter CJ, Smith A and Guillou $P$ (2006): Perception of application of fast track surgical principles by general surgeons. Ann R Coil SurgEngl., 8:191-5.

27. Nanavati A and Prabhakar S (2014): A Comparative Study of 'Fast-Track' Versus Traditional Peri-Operative Care Protocols in Gastrointestinal Surgeries. J Gastrointest Surg., 18:757-76.

28. Proske J, Raue W, Neudecker J, Muller J, Schwenk W (2005): Fast track rehabilitation $\mathrm{m}$ colonic surgery: results of a prospective trial. Ann Chir., 130: 152-6.

29. Hjort $\mathbf{J}$ et al. (2000): A clinical pathway to accelerate recovery after colonic resection. Ann Surg. , 232:51-57

30. Seenu V, Goel AK(1995): Early oral feeding after elective colorectal surgery: Is it safe. Trop Gastroenterol.,16:72-3. 